\title{
Fiber Accelerating Structures
}

\author{
Andrew P. Hammond \\ Office of Science, Science Undergraduate Laboratory Internship Program
}

Reed College

Stanford Linear Accelerator Center

Menlo Park, California

August 14, 2009

Prepared in partial fulfillment of the requirement of the Office of Science, Department of Energy's Science Undergraduate Laboratory Internship under the direction of James E. Spencer in the Advanced Accelerator Research Department at the Stanford Linear Accelerator Center.

Participant:

Signature

Research Advisor:

Signature 


\section{Contents}

1 Introduction $\quad 1$

2 Materials and Methods $\quad 4$

$\begin{array}{lll}3 & \text { Results } & 5\end{array}$

3.1 Simple Fiber Maximization . . . . . . . . . . . . . . . . 5

3.2 Double Defect Modes . . . . . . . . . . . . . . . . . . . . . . . . 7

3.3 Perturbed Periodic Lattice Fibers . . . . . . . . . . . . . . . . . . 9

4 Conclusion $\quad 10$ 


\begin{abstract}
One of the options for future particle accelerators are photonic band gap (PBG) fiber accelerators. PBG fibers are specially designed optical fibers that use lasers to excite an electric field that is used to accelerate electrons. To improve PBG accelerators, the basic parameters of the fiber were tested to maximize defect size and acceleration. Using the program CUDOS, several accelerating modes were found that maximized these parameters for several wavelengths. The design of multiple defects, similar to having closely bound fibers, was studied to find possible coupling or the change of modes. The amount of coupling was found to be dependent on distance separated. For certain distances accelerating coupled modes were found and examined. In addition, several non-periodic fiber structures were examined using CUDOS. The non-periodic fibers produced several interesting results and promised more modes given time to study them in more detail.
\end{abstract}




\section{Introduction}

Currently, linear accelerators use high intensity microwaves to drive the electron bunches through metal radio frequency cavities. Unfortunately, this system is very inefficient. Therefore new systems to better accelerate electrons are being developed. One system that might become a future method for electron acceleration is photonic bandgap (PBG) fibers. It has been shown that PBG fibers could form much better accelerating fibers, if several challenges can be overcome.

Photonic crystals are periodic macroscopic structures that have properties of light propagation similar to those associated with electrical propagation in semiconductors. (Joannopoulos et. all, pg. 4, [3]) One of the key properties for light propagation in photonic crystal is the existence of the photonic bandgap. It is possible using the proper lattice of two dielectric materials periodically spaced to create a band gap in the crystal through which light with frequencies in the bandgap cannot propagate. The theoretical basis for the photonic bandgaps is given by Maxwell's equations of electromagnetism in cgs units. (Joannopoulos et. al, pg. 8, [3])

$$
\begin{array}{r}
\nabla \cdot \mathbf{B}=0 \\
\nabla \cdot \mathbf{D}=4 \pi \rho \\
\nabla \times \mathbf{E}=-\frac{1}{c} \frac{\partial \mathbf{B}}{\partial t} \\
\nabla \times \mathbf{H}=\frac{4 \pi}{c} \mathbf{J}+\frac{1}{c} \frac{\partial \mathbf{D}}{\partial t}
\end{array}
$$

Now, we assume that $\mathbf{D}$ is linearly related to $\mathbf{E}$ by the permitivity $\epsilon(\mathbf{r}, \omega)$ and the permeability is unity so that $\mathbf{B}=\mathbf{H}$. In order to separate the equations from the time domain we assume the solutions take the form

$$
\begin{aligned}
& \mathbf{H}(\mathbf{r}, t)=\mathbf{H}(\mathbf{r}) e^{i \omega t} \\
& \mathbf{E}(\mathbf{r}, t)=\mathbf{E}(\mathbf{r}) e^{i \omega t}
\end{aligned}
$$

This can be done without ignoring solutions because the equations are linear so, Fourier Analysis can be used. In a photonic crystal without free charges or currents, the propagation 
of light is given by the wave equation for dielectrics.

$$
\begin{array}{r}
\nabla \times\left(\frac{1}{\epsilon(\mathbf{r})} \nabla \times \mathbf{H}(\mathbf{r})\right)=\left(\frac{\omega}{c}\right)^{2} \mathbf{H}(\mathbf{r}) \\
\mathbf{E}(\mathbf{r})=\left(\frac{-i c}{\omega \epsilon(\mathbf{r})}\right) \nabla \times \mathbf{H}(\mathbf{r})
\end{array}
$$

Combined with the first two Maxewell equations, this provides the necessary conditions for an electromagnetic wave. In a periodic lattice of dielectrics, there exists the condition of translational symmetry within the problem. If one imagines the dielectric as a series of blocks with period a, then the electric and magnetic fields should be the same a distance $a$ away. Combined with the wave equation the general solution for $\mathbf{H}(\mathbf{r})$ is: (Joannopoulos et. all, pg. 31, [3])

$$
\mathbf{H}(\mathbf{r})=\mathbf{u}_{\mathbf{k}}(\mathbf{r}) e^{i(\mathbf{k} \cdot \mathbf{r})}
$$

This in turn translates to a series of oscillatory modes that light must satisfy to propagate through the structure. However, because the frequency is related to the k-value of light, which defines the wavelength, the light will fluctuate through a series of k-terms as frequency changes to remain within the oscillatory mode. Sometimes, for certain wavelengths the kterm must be imaginary to remain in an oscillatory mode. When the $\mathrm{k}$ is imaginary, it implies light at that frequency exponentially decays due to absorption. In short, light with an imaginary k- term cannot propagate through the crystal.

A useful property of the photonic bandgap is the defect mode. Because the bandgap is caused by the periodic structure of the crystal, when that structure is changed in a localized section, the defect, the allowed modes change as well. In the area surrounding the defect it is possible, depending on the geometry of the defect, for light to propagate even if it has a frequency in the bandgap. However, the defect modes are spatially confined in or near the defect and will not propagate in the rest of the crystal because elsewhere there is still the decay term.

These properties of the PBG crystal can be taken advantage of to accelerate electrons using a PBG fiber. A PBG fiber is an optical fiber consisting of a dielectric material, ideally silicon or silica, with periodic columns of vacuum inside. The fiber used here consists of circular columns used to make a hexagonal fiber. In the fiber the defect is a column with a 
greater radius than the others. The defect column would also serve as the tube through which the electron bunch moves. Lasers would be coupled into the fiber to create electromagnetic waves inside the defect which would accelerate the electrons.

Now, this sort of lattice allows at least two kinds of defect mode: a confined mode and a surface mode (Noble et. all, [7]). In the confined mode the electromagnetic field is confined almost entirely inside the defect column. This mode cannot be used to accelerate particles, but can be used to transmit information i.e. increase longitudinal momentum, because it does not form a uniform axial electric field in the column. Because this mode can transmit information and makes few requirements about precision, it is used by the telecom industry. A surface mode is where the light propagates in the central defect and in the area around the defect where the geometry has changed. In this mode the Poynting vector is concentrated around the surface of the defect column. A surface Poynting vector is shown in Fig. 1; notice the "'hot spots"' and their symmetries in the lattice. Inside of the defect, the surface modes can be uniform, perfect for uniformly accelerating a bunch of electrons. However, surface modes also have other useful field patterns, like the creation of a magnetic quadrupole, or electric lens that could be used to confine the electrons to a tight bunch. Unfortunately, because the electric field exists outside of vacuum, eventually the breakdown field will be reached setting a maximum level on the gradient of the electric field inside of the fiber defect. Therefore, when measuring the electric field a program computes inside a fiber the relevant number is the ratio of the accelerating field to the maximum electric field in the lattice material. Despite this limitation, a PBG accelerator should be several orders of magnitude better than current technology.

In order for these new types of accelerators to be built, several hurdles need to be overcome. Currently the telecom industry uses PBG fibers that support core modes. These fibers are similar in that they come in a lattice and have a defect to those that might be used in an accelerator.(Temelkuran et. all [8]) However, the manufacturers have yet to make a defect mode similar to the one that current designs require. the manufacturers also lack the precision to make the specific modes with a more exact lattice. Also, many of the fiber producers are disinterested in making experimental length PBG fibers, which SLAC would like to test out before ordering longer examples. (Spencer) Fortunately, the Research group 
at SLAC is working with a new company, Incom Inc., to create the necessary fibers. However, they have yet to start using high-purity silica or silicon, or added a defect. The fibers currently possible also require a higher wavelength laser, but such lasers do not exist at all let alone at sufficient power. It is also difficult to find a way to get both the electrons and the laser light coupled into the fiber.

\section{Materials and Methods}

In order to study and understand the electric fields resulting from different PBG geometries, several computer programs were used. The program most used was CUDOS. In addition, Mathematica was used for data analysis, and geometry calculations.

The program CUDOS was built and designed by the Physics department at the University of Sydney in Australia. (Kuhlmey, [5]) CUDOS takes a given geometry and returns the magnetic and electric propagation fields, along with the Poynting vector field. The program uses only a $2 \mathrm{D}$ cross section of the fiber making the field calculation assumption that the fiber has infinite length in the third dimension. The software is based on a method which uses a multipole expansion centered on each cylindrical hole of a fiber to enforce boundary conditions and then matches the local and global expansions to determine the expansion coefficients. (Noble, [6]) Once the various fields have been calculated CUDOS displays a gradient mapping the relative field strength. For a more exact look at the relative fields the raw data was imported to Mathematica.

Once suitable electric field plots were found for various geometries, Mathematica was used to get a more in depth picture of the electric field. While CUDOS does create a relative mapping, the system does not examine the field in a quantitative manner. To look at the subtle field changes, as well as scaling to break-down fields, the plots of the raw data were created using Mathematica.

Mathematica has also been used as a method of calculating the geometries of the lattice. The lattice is arranged in a hexagon with all of the cylinder's centers separated by the same distance from the other. The layers of more hexagons need to be calculated with the position coordinates in the polar system. An example of the cylinder geometry of a CUDOS file is 
found in Fig. 1.

\section{Results}

Several new designs for PBG fibers have been created that are covered in several different sections. One important area is the creation of the best simple fiber by changing the pitch, defect radius, and normal cylinder radius. Also the possible coupling of two defect modes propagating together has been examined. The third area worked on was the effect of nonperiodic symmetry in a PBG fiber.

\subsection{Simple Fiber Maximization}

As mentioned above, the company Incom Inc. has been working with SLAC to create an accelerating PBG fiber. They are currently in the process of creating fibers with defect modes. However, when these new fibers are built they need to be at the proper sizes so that they will accelerate for the lasers that are available. Using the simple designs created by previous students, Eddie Lin, Elliot Johnson, and Sara Campbell, as a starting position; the basic parameters of the hexagonal lattice were modified to find the best simple accelerating fiber. The basic parameters that were modified are the pitch, defect radius, and normal cylinder radius. In this field, the goal was to maximize two parameters, the electric field gradient, and make the defect radius large. It is important to have a high electric field gradient so that the electrons undergo a greater acceleration over a certain distance. It is important to maximize the defect radius so that a larger number of electrons can be fit into the defect radius. As these modes are considered it is important to maintain a uniform field across the defect.

The simple PBG fibers were supposed to be designed for accelerating modes at defect wavelengths of 2, 4, 6, 8, and 16 microns. Now, according to the linear model discussed in the introduction, the wave equation solutions allow a simple scaling of the photonic fiber to increase wavelength. Basically, in a PBG fiber it should be possible to take a fiber with a 2 micron accelerating mode and multiply the basic parameters by 2 to make a 4 micron accelerating mode. (Joannopoulos et. al, pg. 19, [3]) Unfortunately, because the refractive 
index changes a significant amount over the range from 2 to 16 microns, this theory requires parameter adjustment. The refractive index changes as one encounters different energies in which silica is easily excited implying a high level of absorbance. Due to time constraints, only the 2 micron case, which was studied by Sara Campbell and is near the wavelength of the powerful YAG laser, and the 16 micron case, which can already be manufactured, were considered.(Spencer, [1])

The study of the 2 micron PBG fiber started from the fiber design of previous SULI student, Sara Campbell. This design was created by scaling a previous design for a 2.016 micron fiber, as such it had a lerge number of significant figures which I pared down to $10^{-2}$ microns. Once the design was simplified, it was experimented with by making small changes to the pitch, the cylinder center to cylinder center distance, of the fiber. However, this quickly caused the defect mode to stop supporting an accelerating mode at 2 microns. Next, the possible changes that could be made in the normal cylinder radius were examined. The higher the radius of the normal cylinders corresponds to a higher electric field gradient as shown in Fig. 2. Finally the radius of the defect was varied. Unfortunately, the electric field gradient is inversely proportional to the size of the defect mode as shown in Fig. 3. Now in an effort to increase the defect radius regardless of the gradient, the defect radius was increased while lowering the normal radius. Using this approach the maximum defect radius before exiting the bandgap was found to be 1.43 microns. However the field gradient continued to decrease at an even sharper rate, shown in Fig. 4. Unfortunately, the simulation does not work to increase the gradient while decreasing the defect radius and increasing the normal radius.

Now, Mathematica was used to insure that the two fiber designs with maximized defect radius or maximized gradient had a constant longitudinal electric field in the defect. The relevant data for these designs is in Table 1. To make sure the electric field was uniform inside the defect, a graph of the longitudinal electric field along the $\mathrm{x}$-axis and the $\mathrm{y}$-axis was used. The graph was formed from a series of data points output from CUDOS. The graphs and the CUDOS mappings are shown in Fig's. 5, 6, 7, 8, 9 and 10. It is interesting to note that the electric field changes its highest point in the lattice inward as the field gradient becomes larger. The largest defect mode was calculated to have a $3.2 \%$ uniformity 
over the defect, and a $1.9 \%$ uniformity over $80 \%$ of the defect. The defect uniformity over the maximum gradient is $9.8 \%$ and over $80 \%$ of the defect it has a uniformity of $7.7 \%$. The uniformity was calculated by taking the difference between the field at the center of the defect and the maximum field in the defect, and then dividing by the field at the center of the defect. Clearly, although neither plot is completely uniform, the large defect mode is more uniform, possibly because the first high points in the larege defect mode have moved inside of the defect for a smaller defect radius. The lack of uniformity light also imply that several modes are mixed into the calculation.

In addition to the 2 micron defect, a design for a 16 micron wavelength defect was concentrated on. This wavelength was chosen because Incom could make the fibers well, and the index of refraction only changed a small amount from the 2 micron case, allowing the design to be mostly scaled up. When the refractive index changes, the most important change is the size of the defect radius. The change in index most changes the defect radius so with a small change I could concentrate only on this small part of the fiber that is important. The index of refraction increased only by 0.025 from 1.459 to 1.484 (Kitamura, [4]). Using CUDOS, I found the different defect radii that supported acceleration with the new index of refraction. The lowest defect radius value, 11.00 microns is 0.78 microns larger than that scaled from the 2 micron case. This proves the basic point that the defect radius increases for higher index. This result seems to preclude the 8 micron case because its index of 0.407 is so small. However, it might be possible to instead use the small defect to increase the defect size past the first hexagonal ring. The calculated values have been organized in Table 1.

\subsection{Double Defect Modes}

Also the effect that a double defect mode might have on a PBG fiber has been examined. A double defect could have several useful qualities. First, it might be possible to accelerate the electrons in one defect using a coupled defect that is excited by a laser. Also, it could be useful to have several defects inside a single fiber all of which accelerate electrons. This could make up for the fact that only a small number of electrons could fit into the defect cylinder. When examining the multiple defect, only a double defect was examined where 
CUDOS had just two defects. Hopefully, this would reveal any useful coupling or changes in the electric field while not perturbing the coupling by involving multiple defects. In order to concentrate on just coupling the 2 micron accelerating case discussed in the section above was used to specify the basic parameters. However, instead of a single defect cylinder, two defect cylinders were added that had several normal cylinders separating them from one another. The number of cylinders in a straight line between defects was used as the independent parameter specifying the different runs. The defect modes were spaced a range of normal cylinders away from 1 to 9 . Outside this range the number of cylinders lead to leakage of the mode and more could not be added without exceeding the memory capacity of the computer being used.

If the two defects are 1 to 3 cylinders away, the accelerating mode disappears. This is probably because the proximity effects the structure of the bandgap and allows coupling between the defect modes. However, the coupling does not show a useful accelerating mode. For larger distances away, 5 and 7 , the two defects only showed the minor coupling in Fig. 11. This map shows a clear shift in the basic single defect field as shown in Fig. 10. First, even modes that appeared only weakly coupled shifted the points of greatest electric field towards each other as shown in Fig. 11. This might show either coupling or a lack of enough outer cylinders to confine the mode at the outer boundary. When looking a the longitudinal field of the double defect, Fig. 12, the defect field falls off sharply as the distance from the center of the fiber increases. This seems to confirm at least partial loss from the lack of layers. However, by examining the longitudinal field with both 6 and 3 layers of hexagons surrounding the single defect, Fig's. 13 and 14, it is clear that the field is not just the two single modes added together. Even if the modes are coupled, the extent that a beam in one defect could be used to drive a second defect is unknown. Unfortunately, CUDOS does not test this coupling possibility and a more advanced program is necessary. However, it seems worthy of more attention considering the difficulty that the laser coupling already faces. If the two defects were farther apart they lose the accelerating mode and begin to stop holding a mode. However, I suspect this is due to the lack of enclosure by outer cylinders. 


\subsection{Perturbed Periodic Lattice Fibers}

As noted above, the simplest and easiest to construct fibers are those with a single defect and the rest of the cylinders all the same. However, by violating this uniformity it is possible to modify the electric field to accomplish some useful goals. In the attempt to eliminate the hot spots of the fiber, two strategies were used. First, small cylinders of pure vacuum were added on top of the hot spots. However, these small cylinders often failed to cover the entire hot spot, as shown in Fig. 15. The lack of coverage only meant that the side of the cylinder would begin to break down first. The cylinders could not be expanded because then the effective index of refraction would change too much making the wave travel significantly slower than the speed of light. An attempt was also made to eliminate high points in the electric field by expanding certain cylinders while contracting others so that the correct index was maintained. Unfortunately, while this did eliminate the largest high point, all that happened was either other equally strong high points were added which needed to be removed through the small hole method as shown in Fig. 16. The large hole could also significantly perturb the band gap creating new modes that were detrimental to the accelerating structure as shown in Fig. 17. None of these modes is satisfactory to eliminating the hot spots. Currently, I believe that a radically different look of the lattice is necessary to remove the field high points within the lattice. While more work should be done looking for these modes, it is necessary to perform more preparatory analysis to find where to look.

While examining the effect of small hole to eliminate the field high points, it was suggested that the small cylinders could be filled with a new material with a closer index of refraction. If filled with the correct substance, the cylinders could become tiny lasers on the side of the fiber that would drive the electromagnetic field. In a side-laser fiber, several cylinders with different index of refraction simulating the lasing index were added to the CUDOS fiber. In the basic design shown in Fig. 10 there are a series of areas where the field is very high. The goal of this experiment was to keep the high points at the same spot but move them inside cylinders that are filled with a lasing source thus theoretically coupling to drive lasers. These side lasers might be able to then drive the fiber accelerator through natural coupling. One expects this should happen over a short distance because of the need to realign electron 
bunches with the electromagnetic driving wave. The model used YAG as the fill material which has a refractive index of $1.82([2])$. The insertion of minor cylinders with this index had almost no effect on the movement of the accelerating modes longitudinal electric field. Ultimately, all high electric field points were covered by YAG cylinders as shown in Fig. 18 . This is a good sign for the possibility of driving the accelerator in this manner, but it would be very difficult to make. Also there is no guarantee that the lasers would produce the accelerating field and not a new mode. These possible effects depend on the mode of the laser. In addition to driving the accelerating field, the cylinders change the effective index. Although the change is not significant, only a difference of 0.01 , this illustrates the principle that the overall effective index can be changed and controlled. As discussed above this changes the movement of the electromagnetic waves, but it could be useful in re-aligning the electron bunch and the accelerating wave because eventually the wave passes the bunch and starts deaccelerating the bunch.

\section{Conclusion}

A photonic band gap fiber is a promising method for accelerating electrons. There are many clear accelerating modes. that can be built and tested in the near future. There also exist a large number of possible modes that could be examined using the relatively simple program CUDOS to solve the problem of coupling the laser into the fiber. The confining lens is also a viable option that needs more testing, but could be a serious possibility for future fibers. However, a PBG fiber also faces difficulties due to its small size, and the difficulty in coupling laser into the defect mode. Therefore, PBG accelerators are a possible future accelerator source that requires more study. 


\section{References}

[1] Eric Colby, Gary Lum, Tomas Plettner, and James Spencer. Gamma radiation studies on optical materials. IEEE Transactions on Nuclear Science, 49(6), 2002.

[2] Northrop Grumman. Undoped [yag].

[3] John D. Joannopoulos, Robert D. Meade, and Joshua N. Winn. Photonic Crystals, Molding the Flow of Light. Princeton University Press, 1995.

[4] R. Kitamura, L. Pilon, and M Jonasz. Optical constants of fused quartz from extreme ultraviolet to far infrared at near room temperatures. Applied Optics, 46(33):8118-8133, 2007.

[5] Boris Kuhlmey. CUDOS MOF Utilities, User's Guide, 2006.

[6] Robert J. Noble. Dispersion information for photonic fiber modes from cudos simulations. $A R D B$ Note 04, SLAC, 2005.

[7] Robert J. Noble, Eric R. Colby, Benjamin Cowan, Christopher M. Sears, Robert H Siemann, and James E. Spencer. Designing photonic bandgap fibers for particle acceleration. In Particle Accelerator Conference, PAC 0\%, SLAC-PUB-12571, June 2007.

[8] Burak Temelkuran, Shandon D. Hart, Gilles Benoit, John D. Joannopoulos, and Yoel Flink. Wavelength-scalable hollow optical fibres with large photonic bandgaps for co2 laser transmission. Nature, 420, 2002. 


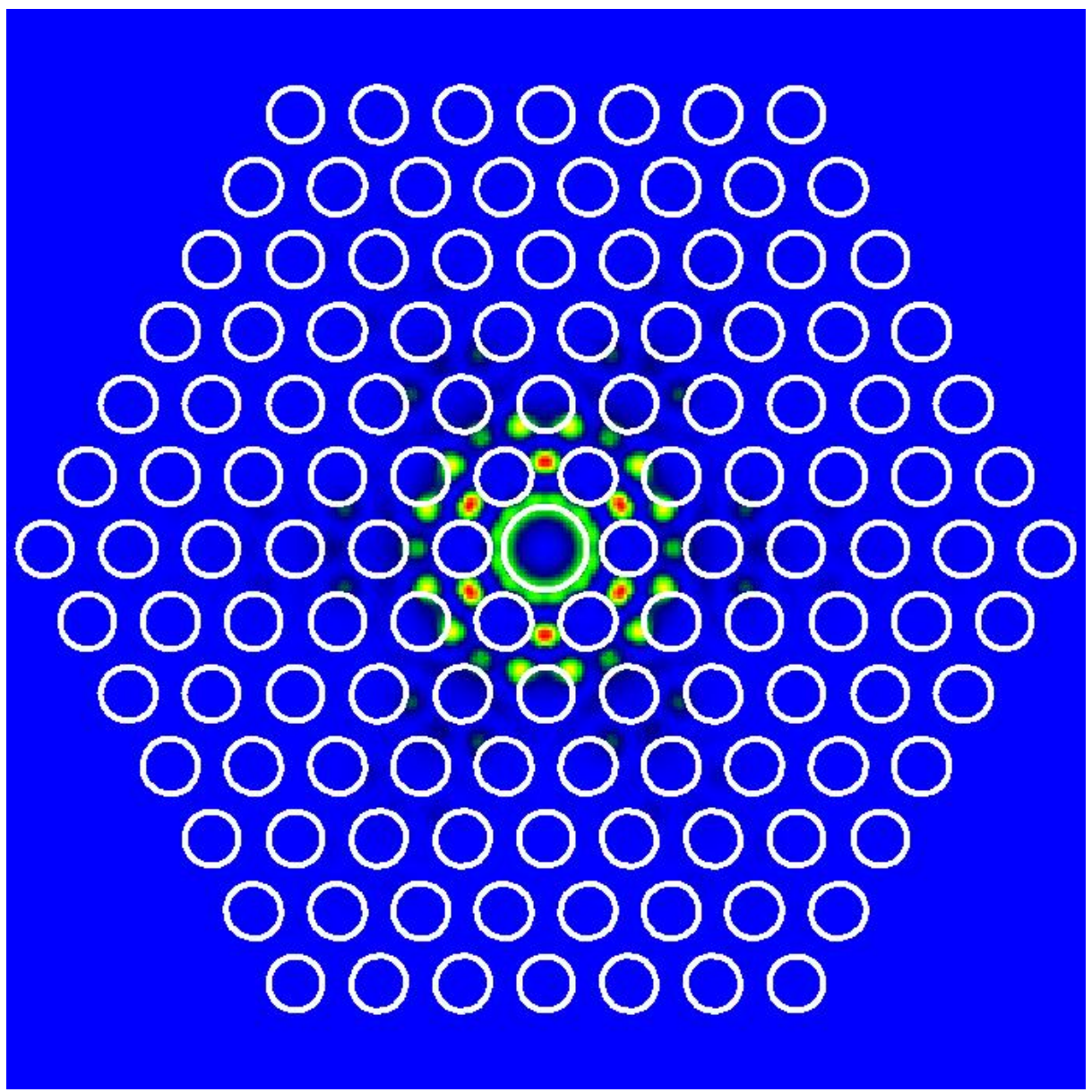

Figure 1: This picture shows the surface mode Poynting vector of a PBG fiber with a defect.

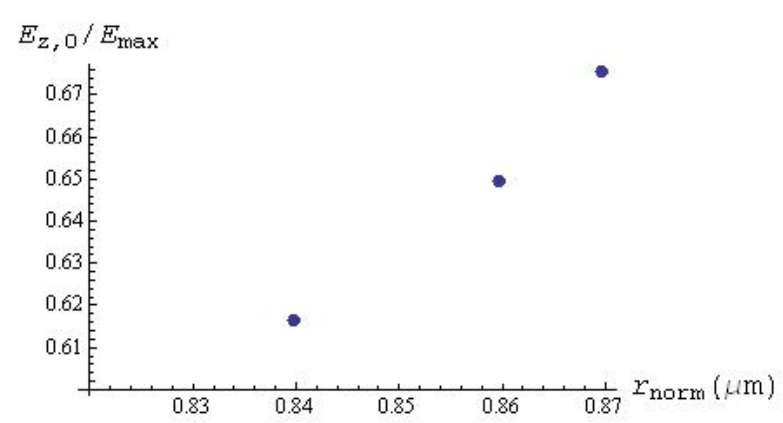

Figure 2: This graph shows the ratio of the longitudinal electric field at the origin to the maximum longitudinal electric field in the fiber(y-axis) compared to the normal cylinder radius(x-axis, measured in microns). The fiber is a 2 micron accelerating fiber the maximum values of which are shown in Table 1 . The ratio is used because it determines the maximum acceleration achievable before the electric field exceeds the fiber's maximum. The plotted points are not all points and there could be smaller radii, the graph just shows the decreasing ratio. 


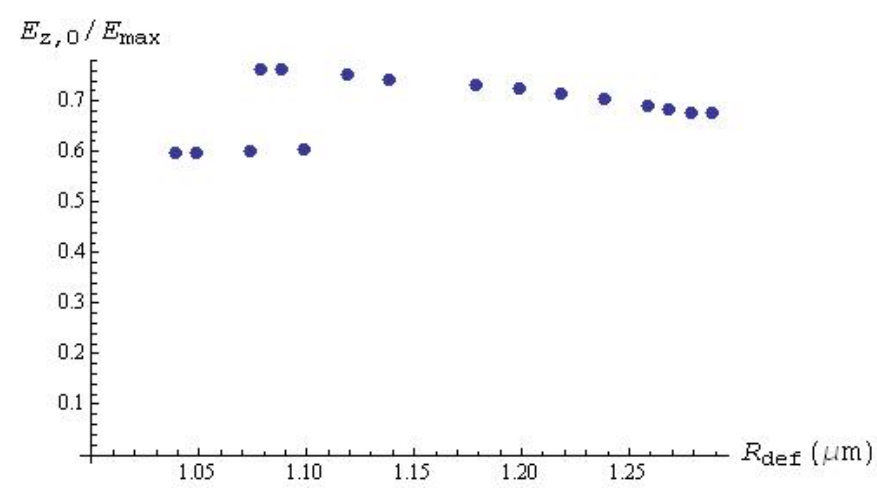

Figure 3: This graph shows the ratio of the longitudinal electric field at the origin to the maximum longitudinal electric field in the fiber(y-axis) compared to the defect cylinder radius(x-axis, measured in microns). The fiber is a 2 micron accelerating fiber the maximum values of which are shown in Table 1 . Near smaller defect radii, the mode begins to switch over into a new ratio regime. This new regime is still an accelerating fiber, but it does not do so as well as the previous regime. This graph shows the entire range of values with a constant normal cylinder radius.

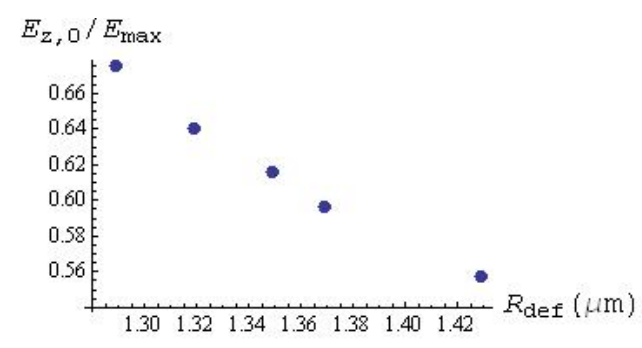

Figure 4: This graph shows the ratio of the longitudinal electric field at the origin to the maximum longitudinal electric field in the fiber(y-axis) compared to the defect cylinder radius(x-axis, measured in microns). The fiber is a 2 micron accelerating fiber the largest defect of which is shown in Table 1. In the graph only the defect radius is shown, but the normal radius is changing as well in order to maintain the accelerating mode. The normal radius each time is as high as it could possibly be consistent with maximum field

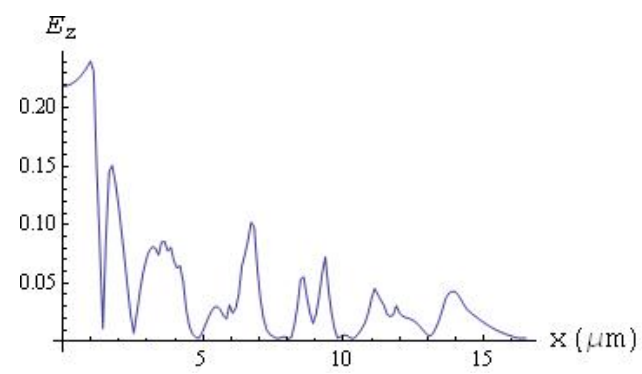

Figure 5: This graph shows the electric field over the x-axis of the highest gradient ratio. The values are shown in row 3 of Table 1. Notice the loss of uniformity around the defect. 


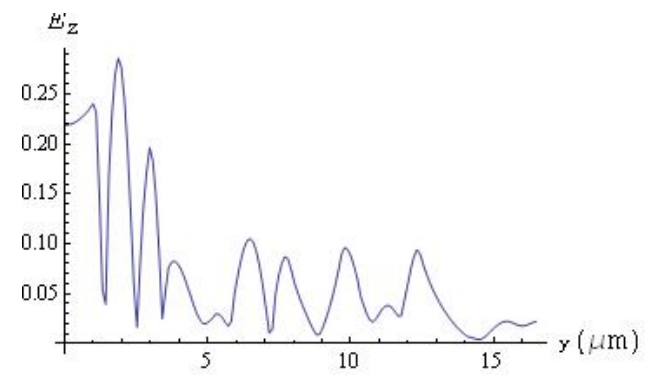

Figure 6: This graph shows the electric field over the y-axis of the highest gradient ratio. The values are shown in row 3 of Table 1. Notice the loss of uniformity around the defect; it is also exactly the same shape inside the defect as in Fig. 5

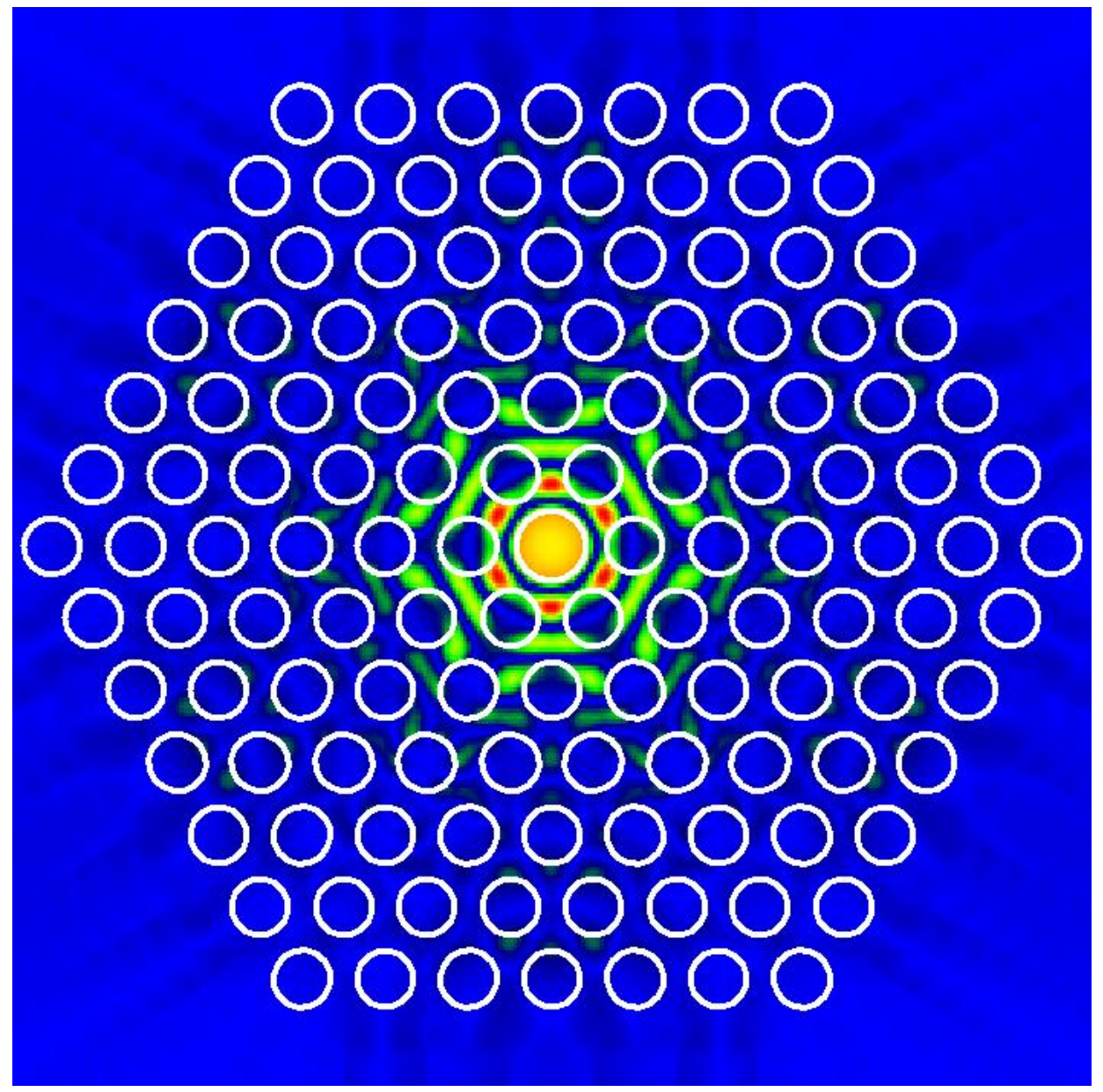

Figure 7: This shows the CUDOS output of the maximum gradient. The values are shown in row 3 of Table 1. 


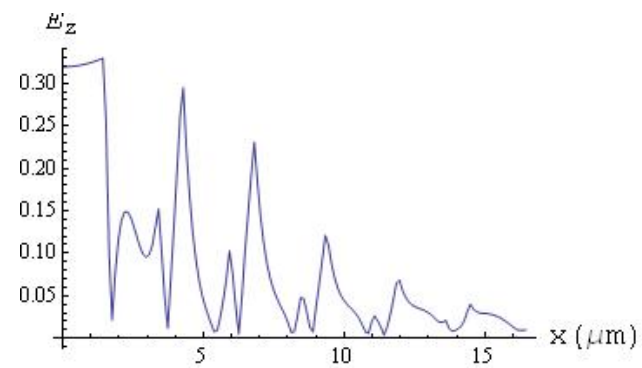

Figure 8: This graph shows the electric field over the x-axis of the highest defect radius. The values are shown in row 2 of Table 1.

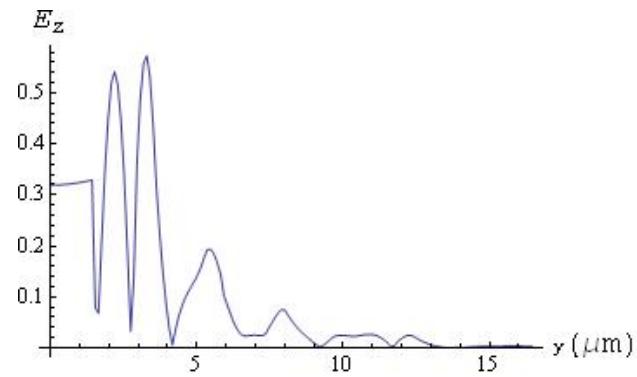

Figure 9: This graph shows the electric field over the y-axis of the highest defect radius. The values are shown in row 2 of Table 1. The defect field here is the same as in Fig. 8.

\begin{tabular}{|c|c|c|c|c|c|c|}
\hline$\lambda(\mu m)$ & $n$ & $n_{\text {eff }}$ & $R(\mu m)$ & $r(\mu m)$ & $P(\mu m)$ & Ratio \\
\hline 2.0 & 1.459 & $1.0017,3.22 \mathrm{E}-4$ & 1.43 & 0.84 & 2.55 & 0.5570 \\
2.0 & 1.459 & $1.0196,1.11 \mathrm{E}-3$ & 1.08 & 0.87 & 2.55 & 0.7639 \\
16.0 & 1.484 & $1.0018,3.67 \mathrm{E}-4$ & 12.50 & 6.92 & 20.40 & 0.4280 \\
16.0 & 1.484 & $1.0192,2.72 \mathrm{E}-4$ & 11.00 & 6.92 & 20.40 & 0.4938 \\
\hline
\end{tabular}

Table 1: This table shows the values of the important variables for the simple lattice structure maximized cases. In the table, $\lambda$ is the wavelength in microns, $n$ is the index of refraction of the matrix, $n_{\text {eff }}$ is the effective index of refraction, a sort of combined refraction for the whole fiber. $R$ is the radius of the defect in microns, $r$ is the normal radius in microns, $P$ is the pitch, the center to center distance, and Ratio is the ratio of the maximum electric field in the lattice to the electric field at the origin. The 2 micron highest gradient case is not the highest tested case because the highest case is in a mixture where two regimes collide. The higher mode might easily be perturbed into a lower gradient mode so a more safe option was chosen. The 16 micron case has also not been tested using a variation of normal cylinder radius, which might improve the maximum values here. 


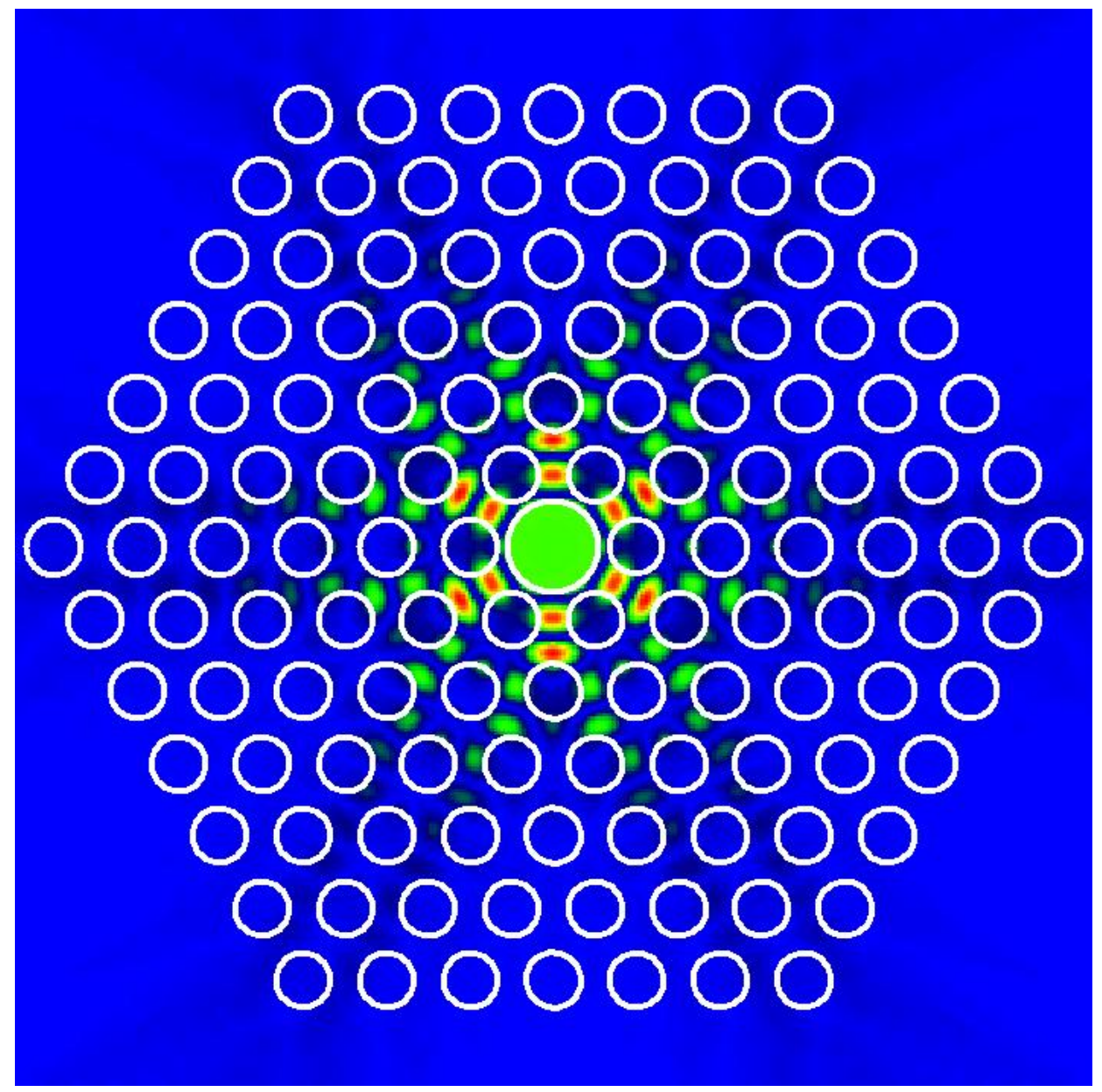

Figure 10: This shows the CUDOS output of the maximum single defect radius. The values are shown in row 2 of Table 1. 


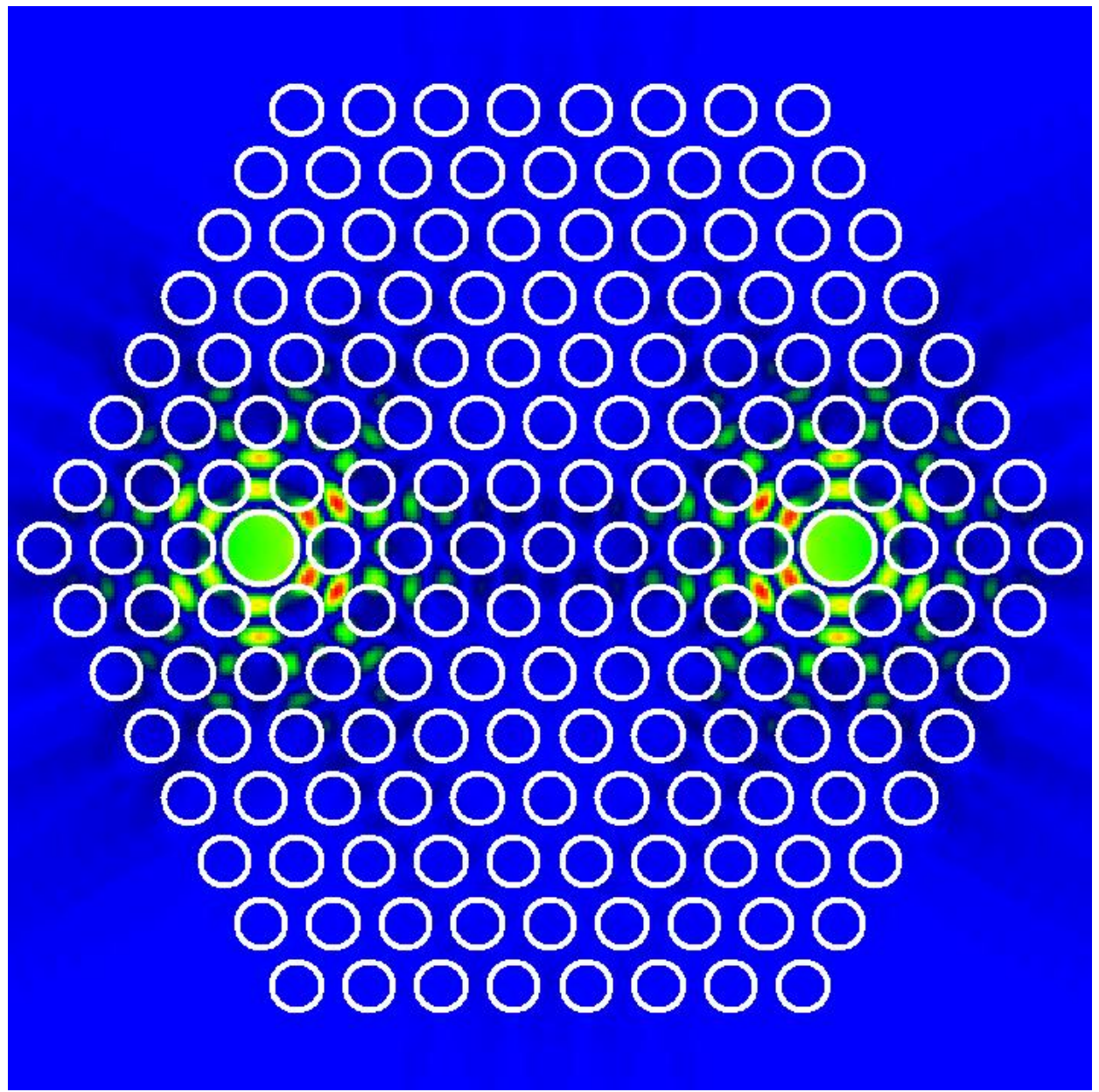

Figure 11: This shows a double defect CUDOS simulation. The values are shown in row 2 of Table 1 except there are two defect radii. Notice the slight coupling along the line of normal cylinders.

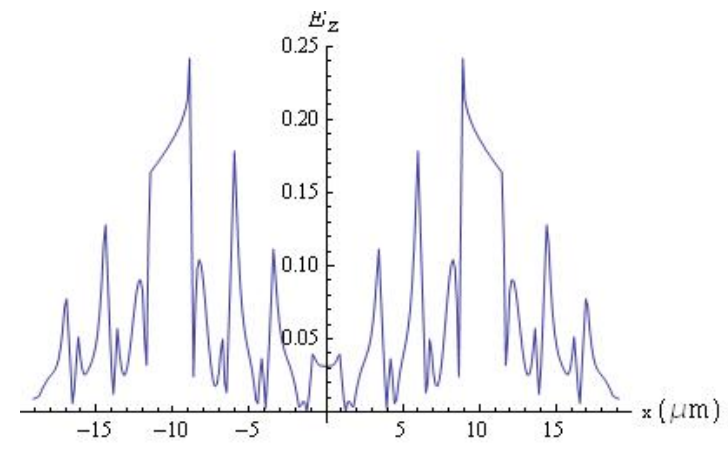

Figure 12: This graph shows the longitudinal electric field along the x-axis. Notice the steep drop off between the two defects extending from \pm 9.65 to \pm 12.19 . However, neither peak is as large as that for a single defect, so perhaps some coupling is occurring. 


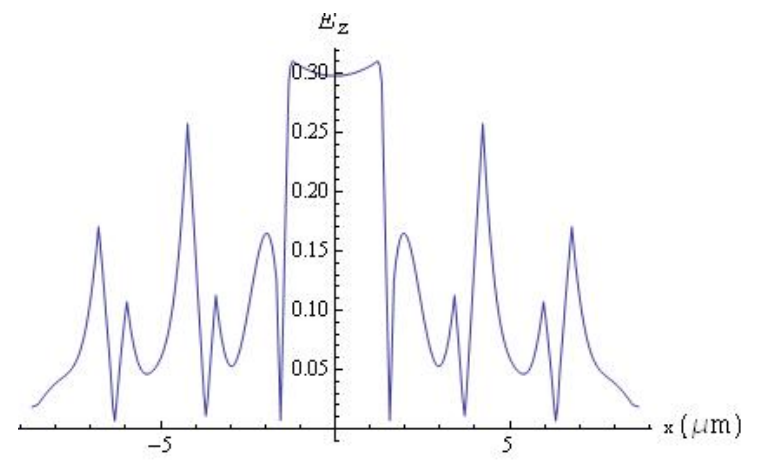

Figure 13: This graph shows the longitudinal electric field for a single defect with only 3 hexagon layers surrounding the defect. The parameters are the same as those for the double defect case, and show a 2 micron accelerating fiber.

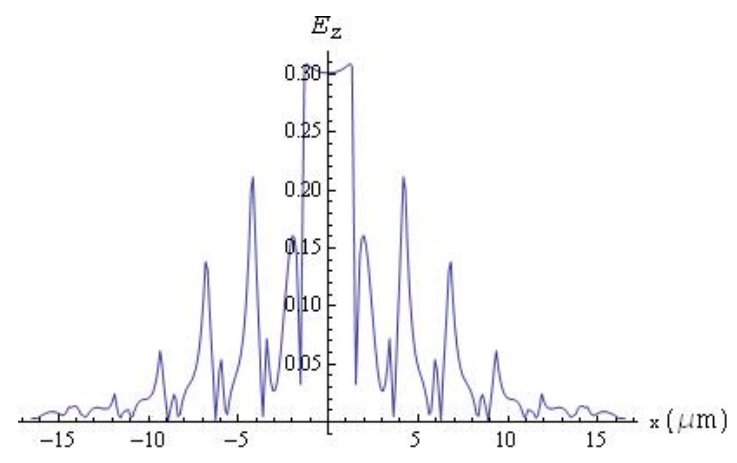

Figure 14: This graph shows the longitudinal electric field for a single defect with 6 hexagon layers surrounding the defect. The parameters are the same as those for the double defect case, and show a 2 micron accelerating fiber. 


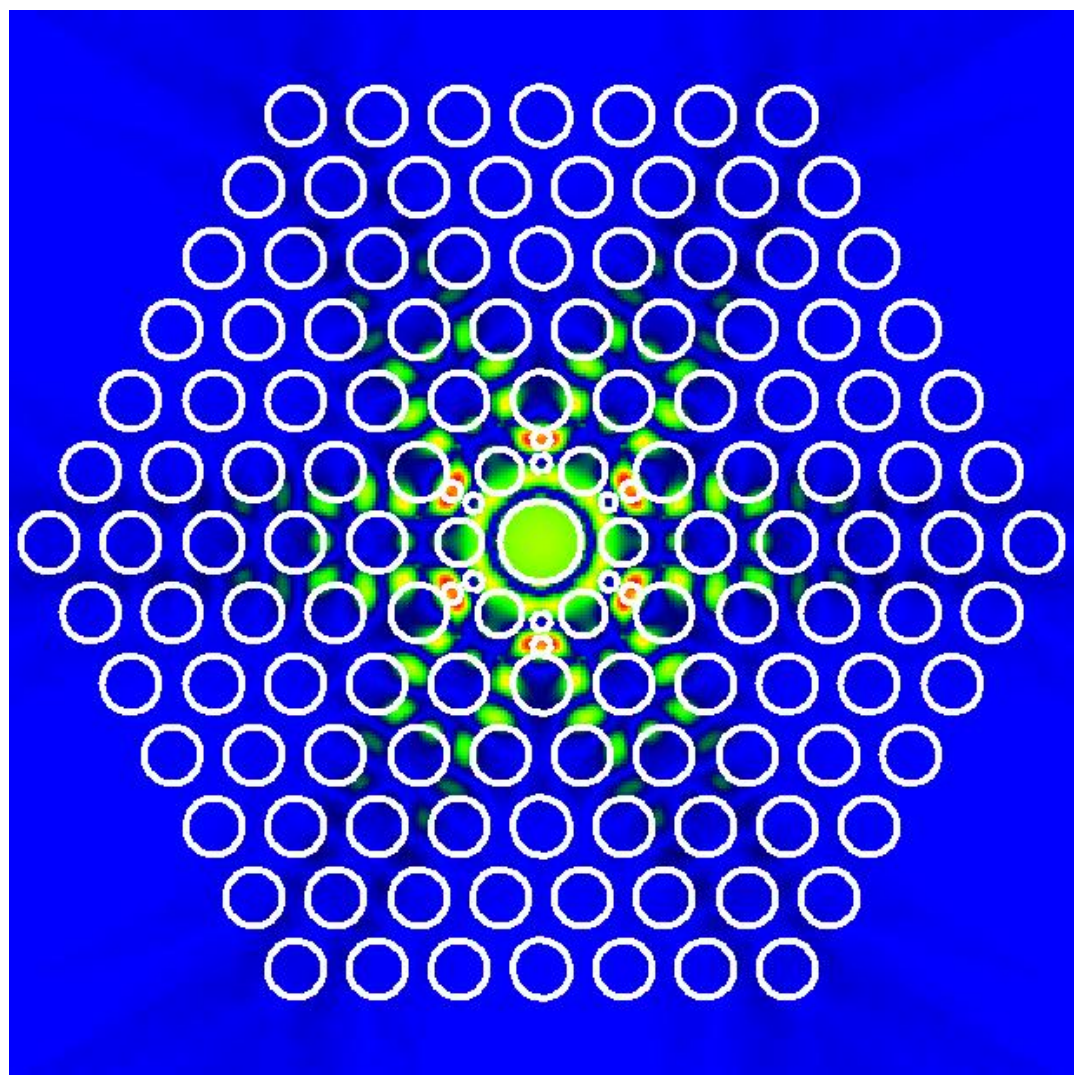

Figure 15: This figure shows the CUDOS map of the longitudinal electric field with small cylinders added to eliminate the hot spots. Notice how the hot spots appear to have expanded from those in Fig. 10. 


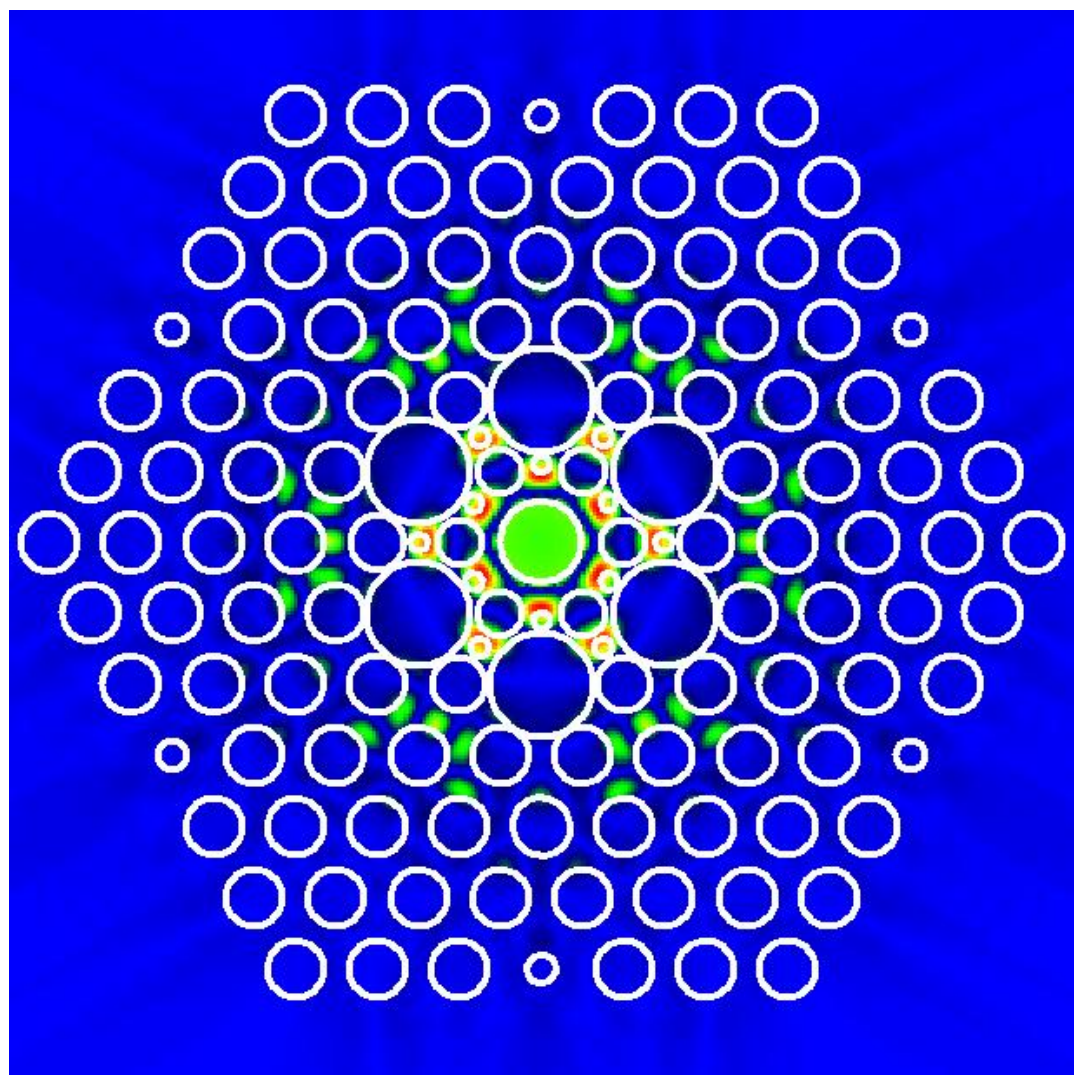

Figure 16: This figure shows the CUDOS map of the longitudinal electric field with a normal cylinder of increased radius to eliminate hot spots. Observe the new hot spots that have been created and covered by small cylinders. 


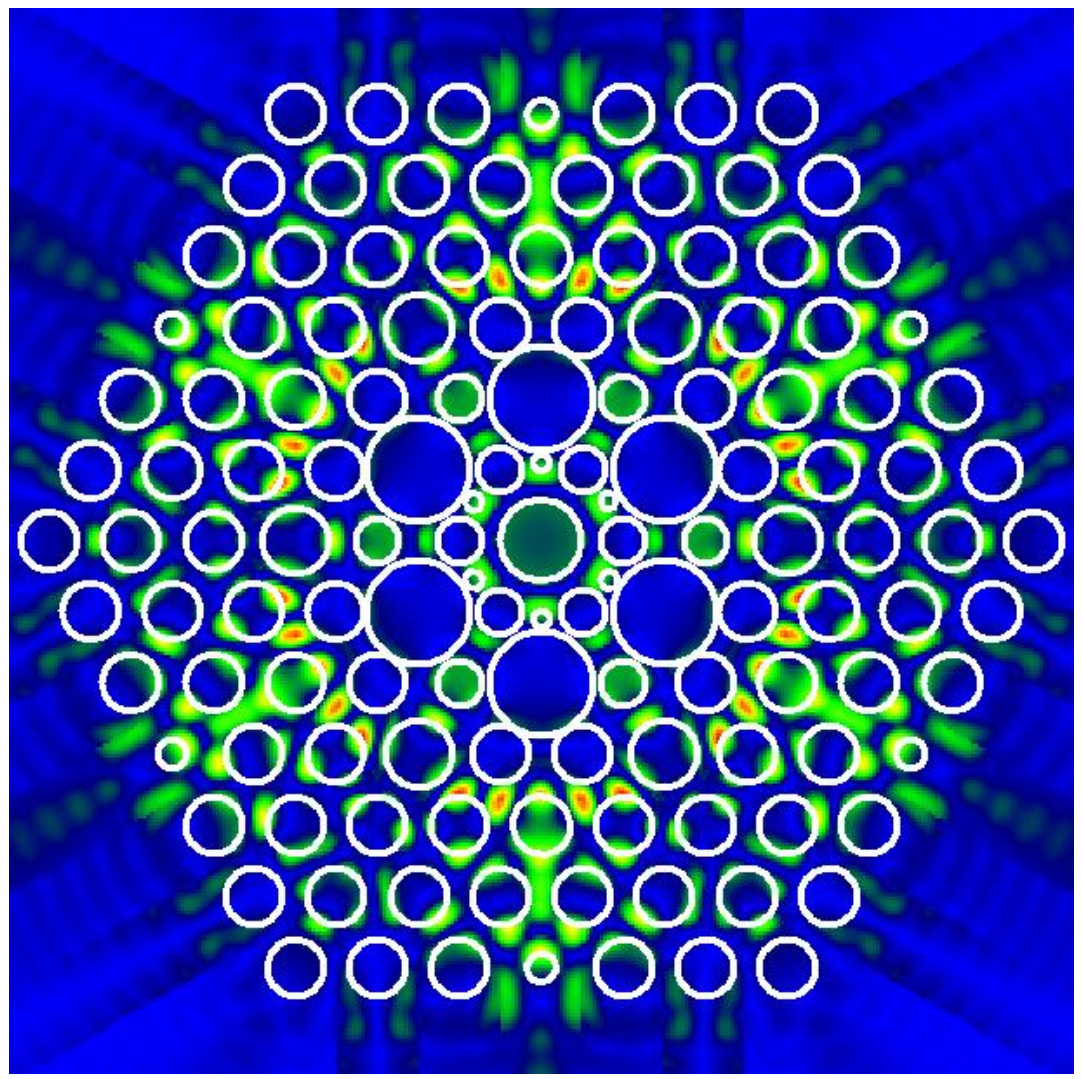

Figure 17: This figure shows the CUDOS map of the longitudinal electric field with a normal cylinder of increased radius to eliminate hot spots. However, the accelerating mode has mostly disappeared and new hot spots have appeared outside of the first hexagons. One now needs to add the missing material back into the lattice to restore the mode. 


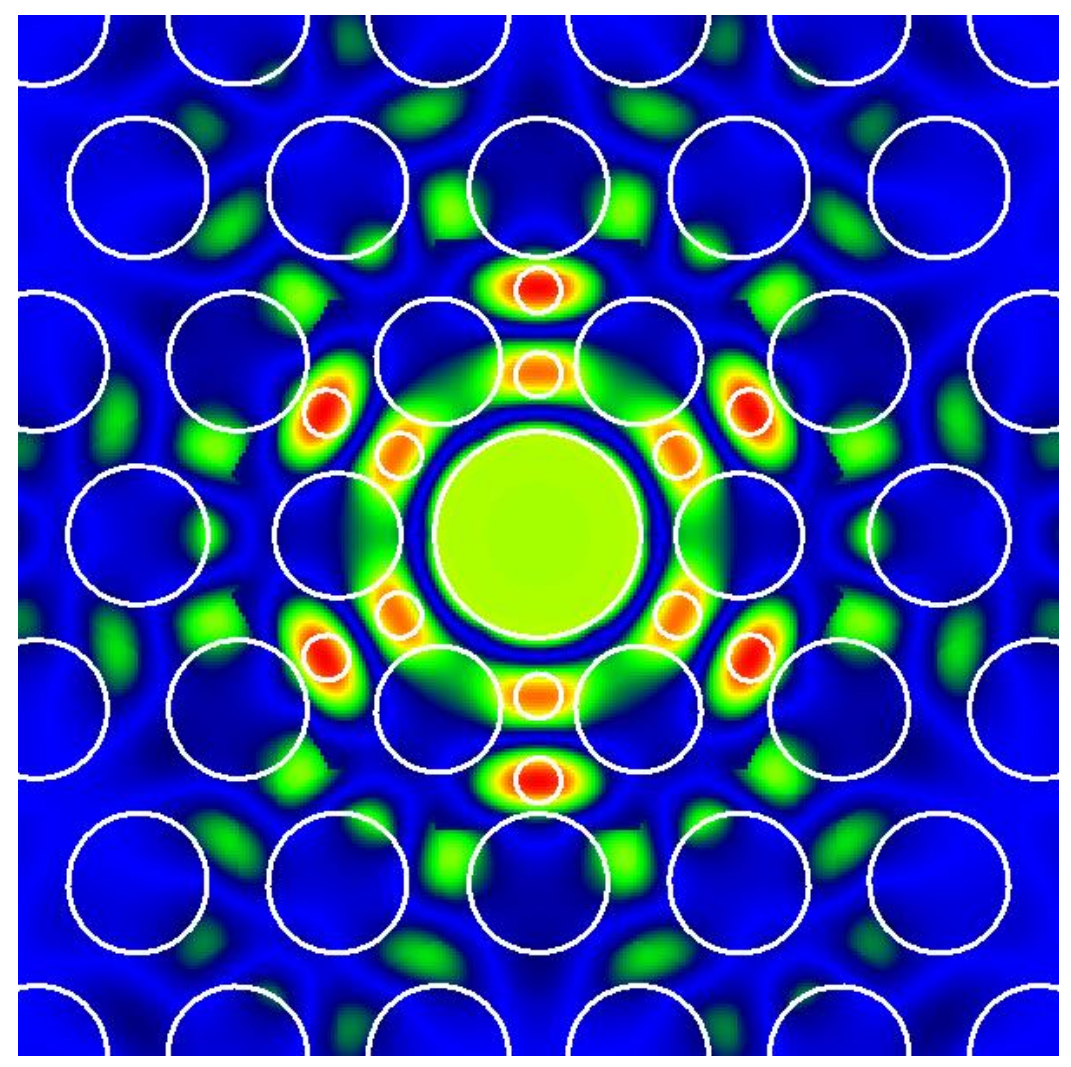

Figure 18: This is a CUDOS file of the longitudinal electric field. I have zoomed in on the central defect to make the smaller cylinders more apparent. The small cylinders have a refractive index equal to that of YAG to make them possible side lasers to drive the electron bunches. Notice that the outer hot spots are strongest. 